\title{
A organização do conhecimento na dinâmica da pesquisa em artigos da literatura científica da Brapci ${ }^{1}$
}

\author{
Knowledge organization in the dynamics of \\ research in the scientific literature \\ of articles at Brapci
}

\author{
Juliana Lazzarotto FREITAS 2 \\ Bruna Silva do NASCIMENTO3 \\ Leilah Santiago BUFREM ${ }^{2}$
}

\section{Resumo}

Este artigo analisa as dimensões de pesquisa expressas concretamente na literatura da área de Organização do Conhecimento. Considera que a produção científica desenvolvida em determinados contextos sociais e momentos históricos reflete as mudanças e contradições desse contexto, tanto em sua organização interna quanto em suas aplicações. Questiona como se configuram as relações entre as opções metodológicas adotadas nessa literatura e o processo dinâmico de pesquisa sob a ótica multidimensional. Ilustra esse processo de análise a partir de um corpus de 105 artigos recuperados da Base Referencial de Artigos de Periódicos em Ciência da Informação, entre 2003 e 2012. Utiliza os softwares Endnote e Excel para organização e análise dos dados coletados. Categoriza os artigos de pesquisa sob o ponto de vista de seu objeto e delineamento metodológico, bem como de suas relações teóricas por meio de estudo de citação. Evidencia os autores mais expressivos e atuantes na área de Organização do Conhecimento no Brasil. Identifica os periódicos mais devotados ao tema e conclui que a produção científica brasileira sobre o tema distribui-se de maneira irregular ao longo do período analisado, tendo seu ápice no ano de 2011, durante o qual são publicados 18,27\% dos artigos. Finaliza estabelecendo relações entre as correntes teóricas da Organização do Conhecimento e os enfoques e temáticas predominantes no corpus. Os enfoques de pesquisa de maior incidência foram o teórico e o linguístico, e o tipo de análise foi documental.

Palavras-chave: Literatura periódica científica. Organização do conhecimento. Pesquisa científica.

\footnotetext{
Abstract

The aim of this paper is to analyze the research dimensions expressed in the literature in the field of knowledge organization. We consider that scientific investigation developed in certain social contexts and historical conjunctures reflects the changes and contradictions present in these contexts, both in its organization and applications. This process is illustrated from a corpus of 105 articles retrieved from

1 Trabalho apresentado no VII Seminário Internacional sobre Estudos Quantitativos e Qualitativos da Ciência e Tecnologia"Prof. Gilberto Sotolongo Aguilar" no XIII Congresso Internacional de Informação - INFO'2014. Habana, Cuba.

2 Universidade Estadual Paulista Júlio de Mesquita Filho, Faculdade de Filosofia e Ciências, Programa de Pós-Graduação em Ciência da Informação. Av. Hygino Muzzi Filho, 737, Mirante, 17525-000, Marília, São Paulo, SP, Brasil. Correspondência para/Correspondence to: L.S. BUFREM. E-mail: <santiagobufrem@gmail.com>.

${ }^{3}$ Universidade Federal do Estado do Rio de Janeiro, Escola de Biblioteconomia, Departamento de Estudose Processos Biblioteconômicos. Rio de Janeiro, RJ, Brasil.

Recebido em 2/6/2014 e aceito para publicação em 20/8/2014.
} 
the referential database of journal articles on Information Science between 2003 and 2012. Endnote and Excel software were used for organizing and analyzing the data collected. The articles are categorized according to their object and methodological approach, as well as their theoretical foundations through the study of citations. Throughout this investigation, we point out the most expressive and active authors in the field of knowledge organization in Brazil. We also identify the journals that are more devoted to the theme and we conclude that Brazilian scientific production on the theme is irregularly distributed over the period of time analyzed, peaking in 2011 when $18,27 \%$ of the total number of articles were published. We conclude by establishing the relationship between theoretical currents of knowledge organization and the prevalent approaches and themes found in the corpus. The focus of the most prevalent research approaches were the theoretical and linguistic approaches and the type of analysis was documentary.

Keywords: Scientific journal literature. Knowledge organization. Scientific research.

\section{Introdução}

A análise de desdobramentos metodológicos das pesquisas em Organização do Conhecimento (OC) no Brasil possibilita a caracterização desse domínio como alternativa para o repensar crítico e autocrítico sobre os meios de construção da pesquisa na área. A percepção da atividade de pesquisa como processo dinâmico, social e histórico - e não somente como recurso para o aprimoramento do fazer científico -, é evidenciada nos argumentos de Lloyd (1995), quando considera que um domínio científico é um recurso para constituir teoricamente objetos de investigação, assim como "para incorporar e fazer justiça à história da ciência em sua acumulação de conhecimento" (Lloyd, 1995, p.25). Com fundamento nas ideias de Lloyd (1995), Bufrem (2013) afirma que a produção científica desenvolvida em determinados contextos sociais e momentos históricos reflete as mudanças e contradições desse contexto, tanto em sua organização interna quanto em suas aplicações.

Em relação à pesquisa retratada no campo da OC, partiu-se de um modelo alternativo e multidimensional para a análise e discussão dos artigos selecionados, ressaltando natureza, gêneros e dimensões relativas à pesquisa. Esse modelo proposto por Bufrem (2013) considera os polos da prática metodológica de Bruyne et al. (1977) como integrantes do processo de pesquisa e os complementa com as dimensões ética e política do processo de investigação científica.

Problematizou-se, neste estudo, como se configuram as relações entre as opções metodológicas adotadas na literatura de OC e o processo dinâmico de pesquisa sob a ótica multidimensional. Como objetivo geral, analisaram-se as opções adotadas na literatura periódica científica de OC sob a ótica do modelo multidimensional da prática de pesquisa. Para tanto, partiu-se da construção de um corpus de artigos selecionado da Brapci a partir de termos relacionados à área. A seguir, categorizaram-se os artigos de pesquisa sob o ponto de vista de seu objeto e delineamento metodológico, bem como de suas relações teóricas representadas por redes de citação. Identificaram-se, então, as opções metodológicas manifestas nessa literatura, relacionando-as com o modelo multidimensional da prática de pesquisa.

Com o intuito de complementar as informações e análises, dentro do contexto da produção e disseminação científica acerca da temática da OC, considerou-se necessário construir um panorama a respeito de quem são os pesquisadores mais profícuos que trabalham com a temática no país. Para tanto, analisaram-se características das autorias, tais como gênero, formação profissional, vínculo institucional, função desempenhada e perfil de publicação desses pesquisadores, destacando-se seus periódicos preferidos para disseminação do conhecimento e, por fim, a modalidade de autoria mais frequente.

\section{Métodos}

Para analisar e relacionar as opções metodológicas do campo de OC no Brasil com as dimensões da pesquisa científica que fundamentam a interpretação aqui realizada, este estudo realizou um recorte de artigos científicos da base de Dados Referenciais de Artigos de Periódicos de Ciência da Informação (Brapci) sobre organização do conhecimento, concernente ao período de dez anos, entre 2003 e 2012, com vistas ao aprofundamento das relações entre os aspectos evidenciados. A Brapci, instrumento para a constituição do corpus, é resultado de um projeto de pesquisa acadêmica com o intuito de facilitar a pesquisa de documentos e artigos da área. Atualmente ela disponibiliza referências e 
resumos de 9673 textos, publicados em 37 periódicos brasileiros, impressos e eletrônicos, da área de Ciência da Informação $(\mathrm{Cl})$, constituindo-se no mais completo repositório da produção científica periódica brasileira em $\mathrm{Cl}$.

Partiu-se de um estudo exploratório na Brapci com os termos descritores "organização do conhecimento", "terminologia" e "conceito" nos campos título, palavra-chave e resumo, obtendo-se um total de 974 artigos. Para atribuir grau de relevância aos registros recuperados empregou-se uma metodologia de pontos incorporada à Brapci, a qual classifica os registros de acordo com a incidência dos termos de busca nesses campos já mencionados, categorizando-se os artigos conforme a relevância para a temática (Freitas et al., 2010). Foram selecionados os artigos que apresentaram ao menos uma estrela cheia para determinar sua relevância, isto é, que continham o descritor em pelo menos dois campos de busca, do que resultou um universo de 177 artigos. Utilizou-se o software Endnote para a organização dos dados coletados, bem como o programa Excel para facilitar a análise dos resultados obtidos.

Em um segundo momento de seleção, foi realizada a leitura dos resumos para descartar pesquisas não pertinentes ao tema em foco, restando um corpus de 105 artigos no período delimitado, considerado significativo, tanto pela diversidade dos estudos que nele se concretizam, quanto pela representatividade de pesquisadores distinguidos no campo de atuação da OC.

É importante salientar que como estratégia de pesquisa utilizou-se a contagem completa dos autores. Ela consiste na atribuição de um valor inteiro para cada um dos autores e coautores. Em decorrência dessa opção, o número total de artigos foi artificialmente aumentado de 105 (títulos diferentes) para 186 (número de autorias). As análises feitas com base no número de autorias foram relativas à formação acadêmica, ao vínculo institucional, à função desempenhada, bem como à produção individual. Acredita-se que as situações relacionadas a essas variáveis são passíveis de alteração ao longo do período analisado. Já para as demais variáveis (temática e opção metodológica, dentre outras) utilizou-se o total de artigos distintos. Essa opção deve-se ao fato de que, dentre as possibilidades de mensurar a colaboração e identificar o perfil dos produtores na área de OC, a contagem completa mostrou-se mais adequada do que a direta, também denominada hierárquica, e a fracionada ou proporcional (Urbizagástegui Alvarado, 2002).

\section{Resultados e Discussão}

Os resultados (Tabela 1) mostram inicialmente que os periódicos mais devotados ao tema OC são DatagramaZero, com 21,90\% dos artigos publicados; Perspectivas em Ciência da Informação, com 12,38\%; Ciência da Informação e Encontros Bibli, cada um com $11,43 \%$ do total de 105 artigos publicados durante o período.

O primeiro lugar no ranking, como veículo de disseminação de trabalhos sobre a OC, obtido pelo periódico DataGramaZero pode ter como justificativa a publicação de um número especial sobre a temática e assuntos circunscritos a ela no ano de 2006. Também foi possível verificar que a maior parte dos artigos foram publicados em revistas pertencentes ao estrato B1 $(61,90 \%)$, sendo que nenhuma produção foi observada em periódicos classificados como A2 na subárea Ciência da Informação, de acordo com o Qualis/Capes.

Dos 114 autores distintos, somente 32,46\% são do sexo masculino, resultado que não diverge de outros estudos de sexo da área de Ciência da Informação, que dão conta de que a maioria dos autores ainda é do sexo feminino. No corpus delimitado apenas um autor não pôde ser identificado (0,88\%), pois somente suas iniciais constavam no artigo e, por não ter nacionalidade brasileira, tampouco faz parte da Plataforma Lattes.

Em relação ao índice de transitoriedade, verifica-se que 84 das 186 autorias publicaram uma única vez no período, resultando em 45,16\% de autores transeuntes. Esse resultado sugere não só uma tendência de consolidação da temática no país, como também indica maior representatividade de alguns que despontam como a elite produtiva sobre a OC.

Quando se aplicou aos 114 autores distintos do corpus a lei do elitismo de Price, que postula que a raiz quadrada do total de autores em determinada área se constitui como elite, obteve-se o número de 10,68 autores situados no topo da lista de produção dentro do tema Organização do Conhecimento (Tabela 2). Entretanto, o 
Tabela 1. Ranking dos periódicos mais profícuos na temática de Organização do Conhecimento (2003-2012).

\begin{tabular}{lccc}
\hline \multirow{2}{*}{ Periódico } & Qualis & \multicolumn{2}{c}{ Contribuição } \\
\cline { 3 - 4 } & & Frequência Absoluta & Frequência Relativa \% \\
\hline DataGramaZero & B1 & 23 & 21,90 \\
Perspectivas em Ciência da Informação & A1 & 13 & 12,38 \\
Ciência da Informação & B1 & 12 & 11,43 \\
Encontros Bibli & B1 & 12 & 11,43 \\
Informação \& Informação & B1 & 8 & 7,62 \\
Transinformação & A1 & 5 & 7,62 \\
Informação \& Sociedade & A1 & 3 & 4,76 \\
Em Questão & B1 & 3 & 2,86 \\
Revista Digital de Biblioteconomia e Ciência da Informação & B1 & 2 & 2,86 \\
Biblos & B3 & 2 & 1,90 \\
InCID & B1 & 2 & 1,90 \\
Ponto de Acesso & B1 & 2 & 1,90 \\
Revista Brasileira de Biblioteconomia e Documentação & B1 & 2 & 1,90 \\
Revista Ibero-Americana de Ciência da Informação & B1 & 2 & 1,90 \\
Tendências da Pesquisa Brasileira em Ciência da Informação & B1 & 1 & 1,90 \\
ACB & B2 & 1 & 0,95 \\
Arquivística.net & B4 & 1 & 0,95 \\
Educação Temática Digital & B4 & 1 & 0,95 \\
Informação e Cognição & B5 & 1 & 0,95 \\
Liinc em Revista & B1 & B1 & 1 \\
Perspectivas em Gestão \& Conhecimento & & 105 & 0,95 \\
\hline Total & & 0,95 \\
\hline
\end{tabular}

Fonte: Dados da pesquisa 2003-2012. Elaboração própria, 2014.

Tabela 2. Autores mais produtivos na área de Organização do Conhecimento (2003-2012).

\begin{tabular}{lcc}
\hline Autor & $\begin{array}{c}\text { Frequência } \\
\text { Absoluta }\end{array}$ & $\begin{array}{c}\text { Frequência } \\
\text { Relativa \% }\end{array}$ \\
\hline MONTEIRO, Silvana Drumond & 7 & 3,76 \\
BUFREM, Leilah Santiago & 6 & 3,23 \\
VAN DER LAAN, Regina & 6 & 3,23 \\
CAMPOS, Maria Luiza de Almeida & 5 & 2,69 \\
KOBASHI, Nair Yumiko & 5 & 2,69 \\
LARA, Marilda Lopes Ginez de & 5 & 2,69 \\
TÁLAMO, Maria de Fátima G. Moreira & 5 & 2,69 \\
GOMES, Hagar Espanha & 4 & 2,15 \\
GUIMARÃES, José Augusto Chaves & 4 & 2,15 \\
BRÄSCHER, Marisa & 4 & 2,15 \\
CAFÉ, Lígia & 4 & 2,15 \\
\hline Total & 55 & 29,57 \\
\hline
\end{tabular}

Fonte: Dados da pesquisa. Elaboração própria, 2014.

recorte utilizado expandiu esse valor para 11 autores, objetivando equalizar o ponto de corte em quatro artigos. Observa-se também que a produção desses autores (29,57\%) não ultrapassa os 50,00\% preconizados por Price para garantir uma elite produtiva.
Em relação à formação e à função desempenhada, não surpreende constatar que $56,45 \%$ dos autores possuem título de doutor e que $63,44 \%$ mencionaram a docência como principal atividade desempenhada. No que concerne ao vínculo institucional, a Universidade de São Paulo (USP) desponta como a primeira colocada com $14,52 \%$, seguida pela Universidade Federal do Rio Grande do Sul (UFRGS) com 10,22\% e pela Universidade Estadual Paulista com 8,06\% das autorias (186).

No intento de melhor compreender esses percentuais, buscaram-se os grupos cadastrados e certificados no último censo de 2010 no Diretório de Grupos de Pesquisa do Conselho Nacional de Desenvolvimento Científico e Tecnológico (DGP/CNPq), utilizando-se as seguintes palavras-chave: organização do conhecimento e organização da informação. Foi possível constatar que, dos 90 grupos revocados, com base nessa estratégia de busca, apenas quatro mencionavam vínculo com a USP, sendo que somente um era da área de Ciência da Informação (Grupo Temma). A partir desse resultado, foi possível identificar que, dos 11 autores componentes da elite, quatro são membros do referido grupo. 
Algumas inferências podem ser feitas com base nesses dados relacionais, especialmente cotejando-os com outros resultados de pesquisas sobre a área de Cl, que mostram uma produção individual ou individualizada. A diferença básica entre esses termos está no fato de que o primeiro se refere à modalidade de autoria, e o segundo, ao processo incipiente de fortalecimento, visibilidade e produção dos grupos de pesquisa. No que concerne à modalidade de autoria dos 105 artigos publicados, observou-se que a maioria foi apresentada na modalidade individual (45,71\%), reforçando estudos anteriores na área de Cl (Mueller \& Pecegueiro, 2001; Vilan Filho, 2010; Nascimento \& Gomes, 2012).

Levou-se em conta, para esta pesquisa, que os fundamentos teóricos de autores relacionam-se com suas posições epistemológicas e políticas, concretizadas nos modos de fazer pesquisa e, por decorrência, nas opções metodológicas eleitas para a realização de suas pesquisas e em suas preferências temáticas. Com esse pressuposto, analisaram-se os tipos de pesquisa quanto aos fins, meios, enfoques e tipos de análises e técnicas eleitos por esses autores no domínio da OC. Para complementar essas posturas metodológicas e também epistemológicas, elencaram-se autores utilizados como suporte teórico pelos autores mais producentes do corpus analisado.

Realizou-se um estudo das referências dos 55 artigos publicados pelo grupo destacado nesta pesquisa para identificar quais autores representam os fundamentos teóricos dos trabalhos analisados. A autora mais citada foi Ingetraut Dahlberg, com 45 incidências (Figura 1). Esse resultado é condizente com a importância das contribuições da autora para a área de Organização do Conhecimento, em especial com a publicação de seu artigo intitulado "Teoria do conceito", de 1978, na revista brasileira Ciência da Informação. Com esse artigo, Dahlberg (1978) estabelece bases teóricas para o entendimento do conceito como estrutura formada por características (enunciados) dos objetos. Essa concepção fundamenta as análises relacionadas à área de Organização do Conhecimento, bem como pode ser relacionada a outros autores basilares, conforme os resultados a seguir.

Ressalta-se que três (30\%) dos autores mais citados pelos mais produtivos são brasileiros, sendo que

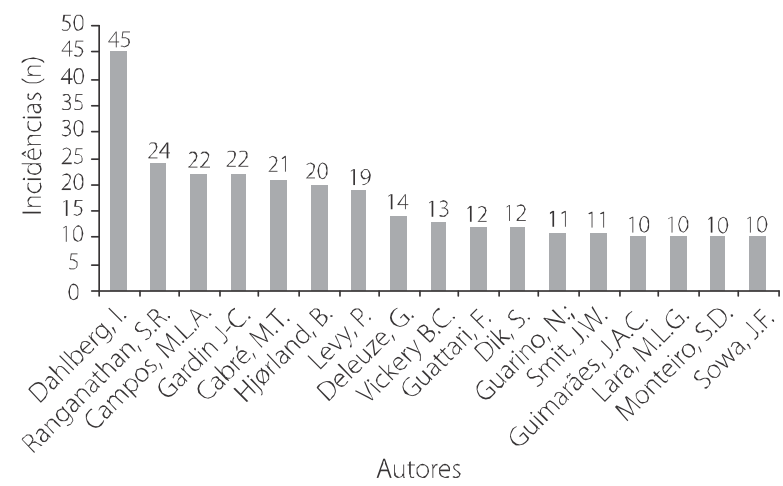

Figura 1. Autores mais citados pelos mais produtivos.

Fonte: Elaboração própria, 2014.

alguns deles também figuram entre os autores mais representativos no corpus. O fato sugere a possibilidade de autocitação, embora o que se tenha verificado é que somente $8,5 \%$ das citações se caracterizaram como tal.

Entre os autores citados concomitantemente pelos mais representativos do corpus (Figura 2), observa-se uma incidência expressiva de Dahlberg com Campos e Dahlberg com Hjorland.

Verificou-se que autores basilares da área de OC figuram entre os mais citados e, em alguns casos, como os mais producentes também. Nesse sentido, uma análise possível seria em relação à presença de artigos voltados à edificação de conceitos teóricos que buscam estabelecer fundamentos do campo.

No que concerne às temáticas consideradas, elas apresentam grande diversidade no que diz respeito às subáreas da Organização e Tratamento da Informação. Sendo assim, optou-se pela descrição somente das que figuram com duas ou mais ocorrências no corpus (Tabela 3).

Ao analisar os dados acima, inferiu-se que a maioria dos trabalhos ( $n=105)$ abordou temáticas não recorrentes (58,10\%), ou seja, esses temas foram trabalhados apenas uma única vez ao longo do período analisado. Dentre as temáticas mais frequentes, a primeira posição é ocupada pela linguagem documentária, e a segunda posição pela terminologia, áreas com presença em atividades diversas relacionadas com a sistematização, representação e edificação de estruturas conceituais que ponderam a lógica e a linguística para seu aprofunda- 


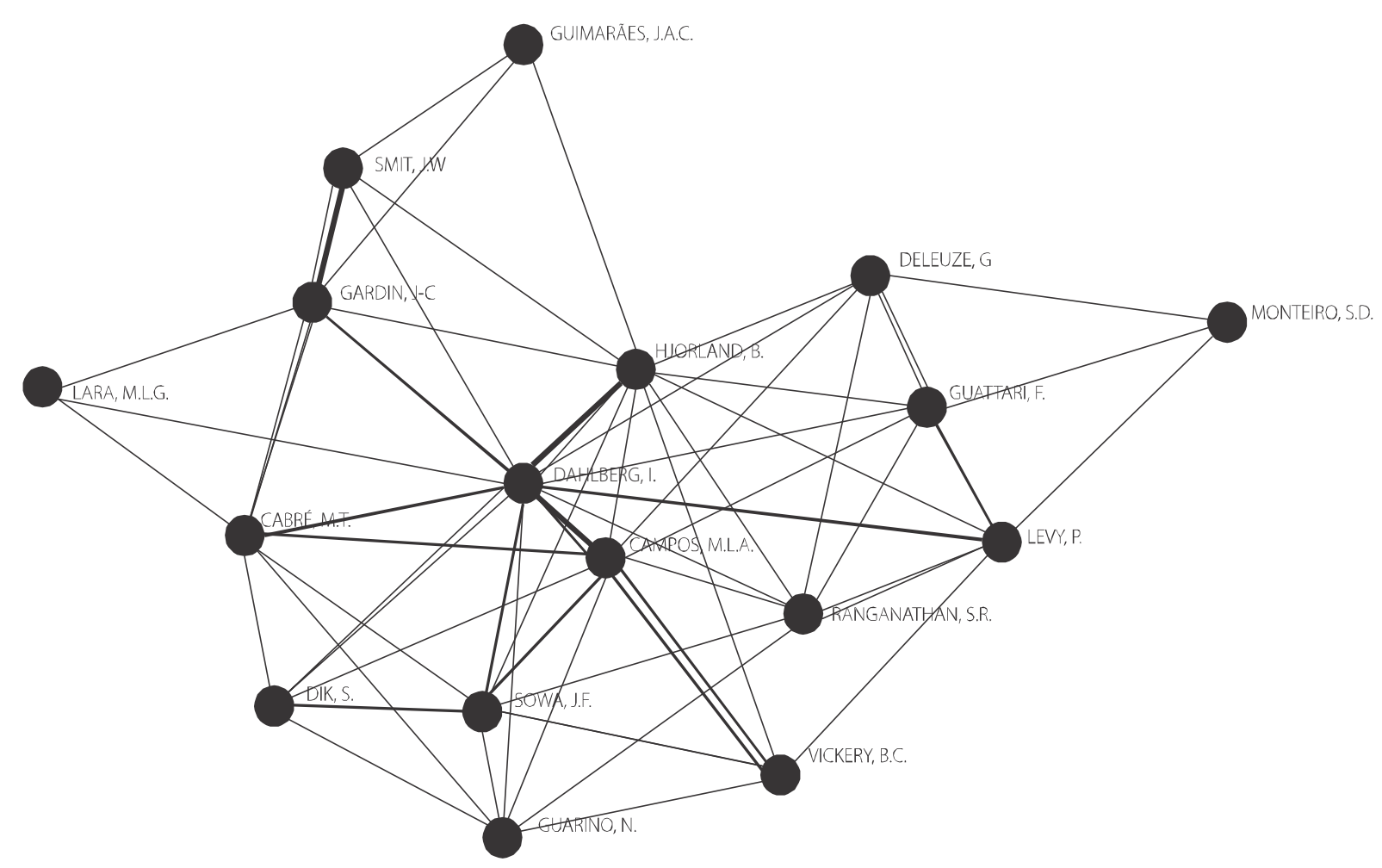

Figura 2. Rede de cocitação dos autores mais representativos do corpus.

Fonte: Elaboração própria, 2014, utilizando o software UCINET.

Tabela 3. Temáticas mais frequentes.

\begin{tabular}{lcc}
\hline \multirow{2}{*}{ Temática } & \multicolumn{2}{c}{ Frequência } \\
\cline { 2 - 3 } & Absoluta & Relativa \% \\
\hline Linguagem Documentária & 16 & 36,36 \\
Terminologia & 11 & 25,00 \\
Conceito & 8 & 18,18 \\
Organização do Conhecimento & 4 & 9,09 \\
Organização Virtual do Conhecimento & 3 & 6,82 \\
Representação do Conhecimento & 2 & 4,55 \\
\hline Total & 44 & 100,00 \\
\hline
\end{tabular}

Fonte: Dados da pesquisa. Elaboração própria, 2014

mento. Os estudos sobre terminologias vão ao encontro das pesquisas relacionadas ao"Tratamento Temático da Informação, ocupando espaço nuclear na literatura e nas práticas profissionais relacionadas à Ciência da Informação [...] uma vez que é por meio dele que se efetiva a mediação entre a produção e o uso da informação, de modo a permitir o acesso ao conteúdo informacional", conforme salientam Guimarães et al. (2012, online). Esse resultado corrobora a adequação teórica dos trabalhos, fato que justifica a presença das autoras Ingetraut Dahlberg (45 incidências) e Maria Tereza Cabré (25) dentre as mais citadas.

As correntes teóricas relacionadas ao Tratamento Temático da Informação, podem ser classificadas, conforme Guimarães (2008) em: catalogação de assunto (subject cataloguing), de influência norte-americana; indexação (indexing), de influência inglesa; e análise documentária (analyse documentaire), de influência francesa.

Segundo Guimarães et al. (2012, online), a análise documental tem como orientação principal a corrente francesa, cujo "[...] foco centra-se no processo de tratamento temático da Informação em si, [...] para a identificação e seleção de conceitos e posterior representação e geração de produtos". Segundo os autores, esse tipo de estudo revela"[...] forte interface com a Lingüística e a Lógica", com especial relevo para a interface da documentação com a linguística (Gardin, 1973) e as questões 
relacionadas às linguagens documentárias (Coyaud, 1966).

Ao identificar as pesquisas em relação aos fins, constata a predominância de pesquisas de caráter exploratório (57,14\%), o que pode indicar não só que a área tem buscado aplicabilidade empírica de suas teorias já sedimentadas, por meio de estudos de caso específicos de organização de informação e conhecimento em unidades de informação organizacionais e em instituições de diferentes tipos, como também aponta para estudos que visam ampliar a familiaridade com os temas propostos.

Em relação aos 34 trabalhos (32,38\%) categorizados como descritivos, salienta-se que esse tipo de estudo se caracteriza por um aprofundamento teórico mais intenso em relação ao objeto estudado, distinguindo-se do que vem sendo observado nos estudos exploratórios, mais incipientes por ensejarem um conhecimento aproximativo das características e aspectos de um objeto de pesquisa. Por sua vez, os estudos descritivos podem representar um amadurecimento na pesquisa teórica da área, por meio do estabelecimento de relações específicas entre variáveis que envolvem as pesquisas. Já os estudos metodológicos, mais complexos, com nove incidências no corpus analisado, geralmente configuramse em propostas de criação de modelos, metodologias e estruturas para a organização da informação em contextos determinados. Em alguns casos, os estudos metodológicos remetem à necessidade de conhecimento sobre tecnologias da informação e comunicação, quando não são exclusivamente modelos conceituais. Nesse caso, exigem-se estudos piloto para validação das propostas. Um exemplo de estudo metodológico no corpus relata modos de estruturação da Base Brapci na Web para acesso universal.

Sobre a última modalidade de pesquisa quanto aos fins, encontram-se os estudos avaliativos, com duas incidências, cujo foco pode ser um objeto, serviço, fluxo, unidade de informação apresentando critérios ou parâmetros a partir de princípios de avaliação. Essas duas últimas tipologias não são expressivas por sua incidência, devido à complexidade de seus processos.

Pode-se verificar que duas das matrizes teóricas relativas às interdisciplinaridades em Análise Docu- mental, postuladas por Guimarães (2010, online) no artigo "Análise documental: concepções do universo acadêmico brasileiro em Ciência da Informação" correspondem à Matriz Quantitativa e à Matriz Tecnológica, que englobam temas relativos à Ciência da Computação e à Informática. Segundo o autor, essas figuram entre as matrizes menos citadas pelos pesquisadores. Entretanto, o "elemento inesperado está na constatação de que a maioria dos pesquisadores relacionou os objetivos da análise documental à Recuperação da Informação, ação que depende diretamente do funcionamento adequado das tecnologias, bem como dos cálculos quantitativos". Esse aspecto, segundo o autor, contradiz a tônica das respostas da pesquisa por ele realizada, e"vai de encontro também com a literatura da área, particularmente nos anos 70 , que tradicionalmente se valeu dos modelos matemáticos para o processo de recuperação da informação" afirma Guimarães (2010, online).

As pesquisas bibliográficas são características da área de $\mathrm{OC}$ e visam estimular seu desenvolvimento conceitual. A predominância desse tipo de pesquisa $(76,19 \%)$ pode reforçar o pensamento de que a institucionalização de qualquer área do conhecimento ocorre mediante a consolidação teórica pela comunidade científica, com o estabelecimento de conceitos, técnicas e metodologias próprias do campo em questão.

Em relação aos enfoques, a maior incidência observada foi o enfoque teórico (49,52\%), seguido pelo enfoque linguístico $(17,14 \%)$, corroborando o exposto acima, já que a maioria dos trabalhos caracterizados como pesquisas bibliográficas apresenta enfoque teórico.

Entre alguns dos autores mais citados, isto é, com mais de três publicações no período estabelecido para esta pesquisa, encontra-se com especial ênfase a preferência ao enfoque teórico, abrangendo estudos relacionados à Filosofia da Linguagem, à Teoria do Conceito, à Teoria da Classificação e à Epistemologia, entre outras correntes filosóficas. O enfoque linguístico engloba temas como Semiologia, Análise do Discurso, Semântica, Linguística Textual, Terminologia, Semiótica, Linguística Documentária, Lógica, Análise de Conteúdo, entre outros, coincidindo com os resultados do estudo de Guimarães (2010, online). Com menor frequência (6,67\%), aparece o enfoque histórico, que considera a 
construção histórica da ciência para a realização de análises.

Em relação ao tipo de análise, a mais frequente foi a análise documental, com 42,67\% dos artigos, seguida pela análise de conteúdo, com 30,67\%. Os demais tipos de análise, como a categorial, a de domínio e a de discurso, obtiveram juntos um percentual menos expressivo (14,67\%) em comparação aos dois primeiros. Outros tipos de análise apresentaram-se de modo ainda mais pulverizado em 7 categorias.

\section{Conclusão}

O presente estudo possibilitou traçar um panorama das publicações na área de $O C$, principalmente no que concerne às características estruturais (opções metodológicas) e estruturantes (autores e autorias) que compuseram o cenário das pesquisas científicas no Brasil nos últimos dez anos (2003-2012).

A presença de autores brasileiros entre os mais citados, mesmo com um percentual de 30\%, reforça a ideia de que comunidade científica brasileira tem buscado articular-se com a comunidade internacional e com autores basilares, acompanhando o desenvolvimento e a evolução do domínio da OC. Sem dúvida, esse fato não prescinde da inquestionável importância dos autores internacionais para sua constituição teórica enquanto área de pesquisa, assim como das influências das distintas correntes de Organização e Tratamento Temático da Informação para o desenvolvimento e enriquecimento da OC em âmbito nacional. Entretanto, não se pode deixar de destacar o amadurecimento dos pesquisadores nacionais e a constituição de laços colaborativos entre eles.

\section{Referências}

Bruyne, P. et al. Dinâmica da pesquisa em ciência sociais: os polos da prática metodológica. 2.ed. Rio de Janeiro: F. Alves, 1977.

Bufrem, L.S. Configurações da pesquisa em ciência da informação. DataGramaZero: Revista de Informação, v.14, n.6, 2013. Disponível em: <http://www.dgz.org.br/ago14/ F_l_aut.htm>. Acesso em: 9 ago. 2014.

Coyaud, M. Introduction à l'étude des langages documentaires. Paris: Klinsieck, 1966.
A realidade observada depõe em favor da construção de um ethos próprio da OC no país, pois é inquestionável que a institucionalização de qualquer área do conhecimento ocorre mediante sua consolidação teórica pela comunidade científica, com o estabelecimento de conceitos, técnicas e metodologias que a distingam das demais. Essa construção se caracteriza por ser uma edificação realizada pelos e entre os agentes envolvidos, os quais estabelecem relações, práticas e preceitos ético-políticos identificados como exclusivos de uma comunidade. Cumpre ressaltar que aqui se considera o conceito de comunidade como revelador de uma identidade individualizante e distintiva, mas aglutinadora. Isso significa que, para pertencer ou transitar em determinada área, é imprescindível que se partilhe desse ethos que se constitui das e nas práxis empreendidas pelos membros que privam não só desse código e objetivo comum, mas também buscam o reconhecimento e a expansão da área perante a comunidade científica.

Por fim, ressalta-se que uma futura pesquisa, que estabeleça as relações de citação, bem como identifique a colaboração intra e interinstitucional, presentes no corpus analisado, pode esclarecer ainda mais de que maneira a área tem se sedimentado ao longo dos anos. Além disso, será possível verificar sob quais aspectos epistemológicos a OC se edifica e se torna reconhecida pela Ciência da Informação.

\section{Agradecimentos}

Trabalho apoiado por concessão de bolsa da Fundação de Amparo à Pesquisa do Estado de São Paulo (Fapesp).

Dahlberg, I. A referent-oriented analytical concept theory of interconcept. International Classification, v.5, n.2, p.142-150, 1978.

Freitas, J.L. et al. Proposta de metodologia para a recuperação da produção científica em ciência da informação na base Brapci. Ponto de Acesso, v.4, n.3, p.45-67, 2010.

Gardin, J.-C. Document analysis and linguistic theory. Journal of Documentation, v.29, n.2, p.137-168, 1973. 
Guimarães, J.A.C. A dimensão teórica do tratamento temático da informação e suas interlocuções com o universo científico da International Society for Knowledge Organization (ISKO). Revista Ibero-americana de Ciência da Informação, v.1, n.1, p.77-99, 2008.

Guimarães, J.A.C. Análise documental: concepções do universo acadêmico brasileiro em ciência da informação. DataGramaZero, v.1, n.1, 2010. Disponível em: <http://www. dgz.org.br/fev10/Art_02.htm>. Acesso em: 9 ago. 2014.

Guimarães, J.A.C et al. A dimensão interdisciplinar da análise documental nos contextos brasileiro e espanhol no âmbito da organização do conhecimento. DataGramaZero, v.13, n.6, 2012. Disponível em: <http://www.dgz.org.br/dez12/ Art_07.htm>. Acesso em: 9 ago. 2014.

Lloyd, C. As estruturas da história. Rio de Janeiro, Zahar, 1995.
Mueller, S.; Pecegueiro, C.M.P.A. O periódico ciência da informação na década de 90: um retrato da área refletido em seus artigos. Ciência da Informação, v.30, n.2, p.47-63, 2001

Nascimento, B.S.; Gomes, M.Y.F.S.F. A ciência da informação no Brasil: um retrato da área através do estudo de autoria. In: Encontro Nacional de Pesquisa em Ciência da Informação, 13., 2012, Rio de Janeiro. Anais eletrônicos..., Rio de Janeiro: Associação Nacional de Pesquisa e Pós-Graduação em Ciência da Infor-mação, 2012. p.1-21. Disponível em: <http:// www.eventos econgressos.com.br/metodo/enancib2012/ arearestrita/pdfs/19487.pdf>. Acesso em: 9 ago. 2014.

Urbizagástegui Alvarado, R. A lei de Lotka na bibliometria brasileira. Ciência da Informação, v.31, n.2, p.14-20, 2002.

Vilan Filho, J. Autoria múltipla em artigos de periódicos científicos das áreas de informação no Brasil. 2010. Tese (Doutorado em Ciência da Informação) - Departamento de Ciência da Informação e Documentação, Universidade Federal de Brasília, Brasília, 2010 

